\title{
EFFECTIVE ALGORITHM FOR DETERMINATION OF THE INITIAL VeCTOR FOR THE DEFINITE QUADRATIC PENCIL
}

\author{
Aleksandra Kostic, Zajim Aljicevic, Sefko Sikalo \& Melisa Kustura
}
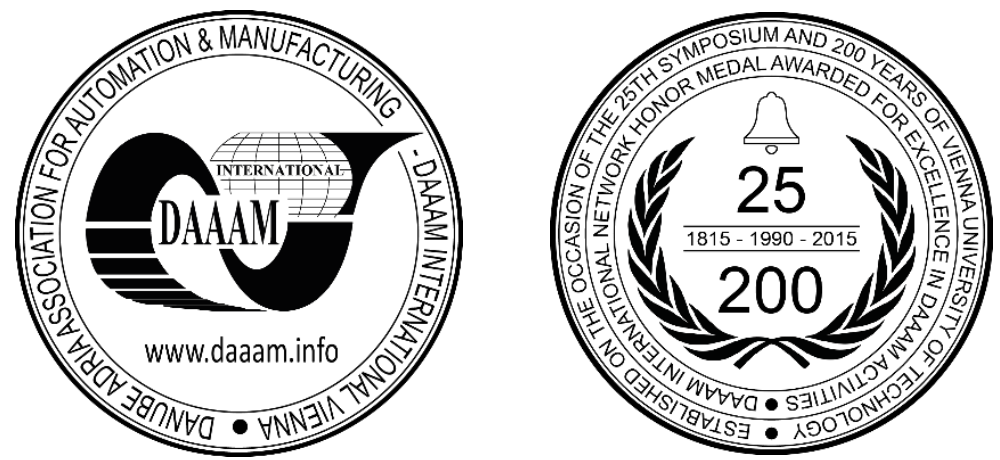

This Publication has to be referred as: Kostic, A[leksandra]; Aljicevic, Z[ajim]; Sikalo, S[efko] \& Kustura, M[elisa] (2016). Effective Algorithm for Determination of the Initial Vector for the Definite Quadratic Pencil, Proceedings of the 27th DAAAM International Symposium, pp.0010-0016, B. Katalinic (Ed.), Published by DAAAM International, ISBN 978-3-902734-08-2, ISSN 1726-9679, Vienna, Austria

DOI: $10.2507 / 27$ th.daaam.proceedings.002

\begin{abstract}
In this paper we study properties of definite quadratic eigenproblems. Free vibrations of fluid-solid structures are governed by a nonsymmetric eigenvalue problem. This problem can be transformed into a definite quadratic eigenvalue problem. Niendorf and Voss presented an algorithm which determents if quadratic pencil is or is not definite. In their algorithm, the initial vector is taken at random. In this paper we bring a new effective algorithm for determining the initial vector $\mathbf{x}_{0}$ and thus we accelerate algorithm of Niendorf and Voss. The new algorithm presented in this paper combines earlier results of Kostic and Sikalo with the new results obtained in this study.
\end{abstract}

Keywords: free vibrations; definite quadratic pencil; eigenvalue; methods for detection; effective algorithm; initial vector.

\section{Introduction}

In the engineering practice eigenvalue problem is very important because it is related with the mechanical systems and their property of vibration. Vibration is property of every mechanical system. Also it is property of electrical systems in the form of oscillation circuits. Neglecting terms of vibration can lead to resonance. The resonance can have catastrophic consequences (for example the demolition of the bridge), on the other hand it can be used positively. Mathematical description of vibratory conditions leads to a differential equation or a system of differential equations. This problem is further transformed to the eigenvalue problem. This is the motivation for considering the eigenvalue problems. They are divided into linear and nonlinear problems of eigenvalues. The theoretical basics for eigenvalue problems are given in the [7]. In practice, nonlinear eigenproblems commonly arise in dynamic/stability analysis of structures and in fluid mechanics, electronic behavior of semiconductor hetero-structures, vibration of fluid-solid structures, regularization on total least squares problems and stability of delay differential equations.

In practice, most important is the quadratic problem $\mathbf{Q}(\lambda) \mathbf{x}=\mathbf{0}$ where

$$
\mathbf{Q}(\lambda):=\lambda^{2} \mathbf{A}+\lambda \mathbf{B}+\mathbf{C}, \quad \mathbf{A}, \mathbf{B}, \mathbf{C} \in \mathbb{C}^{n \times n}, \quad \mathbf{A} \neq \mathbf{0},
$$

More information on the practical application can be found in [12]. 
Since we, in this paper, deal with the improving of the algorithm to determine whether a quadratic eigenvalue problem is definite or not, let us briefly summarize what has been done so far in the literature up to this point.

For quadratic hyperbolic pencils, Higham, Tisseur and Van Dooren [5] proposed a method for testing hyperbolicity and for constructing a definite linearization. Another method for detecting whether a Hermitian quadratic matrix polynomial is hyperbolic is based on cyclic reduction and was first introduced by Guo and Lancaster [3] and later on, accelerated by Guo, Higham and Tisseur [2].

Niendorf and Voss [10] take advantage of the fact that all eigenvalues of a definite matrix polynomial can be characterized as minmax values of the appropriate Rayleigh functional and that the extreme eigenvalues in each of the intervals $(-\infty, \xi),(\xi, \mu)$ and $(\mu,+\infty)$ are the limits of monotonically and quadratically convergent sequences. If a given Hermitian quadratic matrix polynomial is definite, Niendorf and Voss concurrently determine parameters $\xi$ and $\mu$ such that the matrices $\mathbf{Q}(\xi)<0$ and $\mathbf{Q}(\mu)>0$, which allows for the computation of a definite linearization.

Kostic and Voss [9] applied the Sylvester's law of inertia on the definite quadratic eigenvalue problems.

In paper [8] Kostic and Sikalo gave improvement of the method of Niendorf and Voss [10] for detecting whether a quadratic pencil $\mathbf{Q}(\lambda)$ is definite. They have used some properties of matrices $\mathbf{A}, \mathbf{B}$ and $\mathbf{C}$ to get improvment of the algorithm. These properties are rank of the matrices $\mathbf{A}, \mathbf{B}$ and $\mathbf{C}$ and eigenvectors of these matrices which corespond to the egenvalue zero. Kostic and Sikalo in [8] obtained good results when matrix $\mathbf{B}$ is singular. If matrix $\mathbf{B}$ is not singular they would have to determine eigenspace of the matrix $\mathbf{A}$, to the eigenvalue zero. Also, they would have to determine eigenspace of the matrix $\mathbf{C}$, to the eigenvalue zero. This reduces efficiency of their idea.

This paper gives effective algorithm for determination of the initial vector $\mathbf{x}_{0}$. The algorithm is based on results of the paper [8] and on application of eigenvector $\mathbf{q}_{\mathbf{1}}$ which is corresponding vector of eigenvalue $\lambda_{\mathbf{1 A}}$ of matrix $\mathbf{A}$, and also on application of eigenvector $\mathbf{f}_{\mathbf{n}}$ which is corresponding vector of eigenvalue $\lambda_{\mathbf{n} \mathbf{B}}$ of matrix $\mathbf{B}$.

Our paper is organized as follows. In Section 2 we provide definition of the definite eigenvalue problem and results of previous papers based on this topic. Section 3 gives a new effective algorithm for determining of the initial vector $\mathbf{x}_{0}$. This section also presents new results which lead to our algorithm. A critical review of the proposed algorithm and the future research plan is given in Section 4.

\section{Definite quadratic pencils}

In this paragraph we give the theoretical basics for the definite quadratic pencil. Since the definite quadratic polynomial is generalization of the hyperbolic quadratic polynomial, let us briefly consider the hyperbolic quadratic polynomial.

A quadratic matrix polynomial

$$
\mathbf{Q}(\lambda):=\lambda^{2} \mathbf{A}+\lambda \mathbf{B}+\mathbf{C}, \quad \mathbf{A}=\mathbf{A}^{H}>0, \quad \mathbf{B}=\mathbf{B}^{H}, \quad \mathbf{C}=\mathbf{C}^{H}
$$

is hyperbolic if for every $\mathbf{x} \in \mathbb{C}^{n}, \mathbf{x} \neq \mathbf{0}$ the quadratic polynomial

$$
\mathbf{f}(\lambda ; \mathbf{x}):=\lambda^{2} \mathbf{x}^{H} \mathbf{A} \mathbf{x}+\lambda \mathbf{x}^{H} \mathbf{B} \mathbf{x}+\mathbf{x}^{H} \mathbf{C x}=0
$$

has two distinct real roots:

$$
p_{+}(\mathbf{x}):=-\frac{\mathbf{x}^{H} \mathbf{B x}}{2 \mathbf{x}^{H} \mathbf{A x}}+\sqrt{\left(\frac{\mathbf{x}^{H} \mathbf{B x}}{2 \mathbf{x}^{H} \mathbf{A x}}\right)^{2}-\frac{\mathbf{x}^{H} \mathbf{C x}}{\mathbf{x}^{H} \mathbf{A x}}}, \quad p_{-}(\mathbf{x}):=-\frac{\mathbf{x}^{H} \mathbf{B x}}{2 \mathbf{x}^{H} \mathrm{Ax}}-\sqrt{\left(\frac{\mathbf{x}^{H} \mathbf{B x}}{2 \mathbf{x}^{H} \mathrm{Ax}}\right)^{2}-\frac{\mathbf{x}^{H} \mathbf{C x}}{\mathrm{x}^{H} \mathrm{Ax}}}
$$

Functionals in (4) are the Rayleigh functionals of the quadratic matrix polynomial (2). The Rayleigh functionals are the generalization of the Rayleigh quotient.

The hyperbolic problem is very importance because all of its eigenvalues can be variational characterized. More information is contained in [1].

Higham, Mackey and Tisseur [6] generalized the concept of the hyperbolic quadratic polynomial by waiving the positive definiteness of the leading matrix $\mathbf{A}$.

\section{Definition 1}

A quadratic pencil (1) is definite if:

$\mathbf{A}=\mathbf{A}^{\boldsymbol{H}}, \mathbf{B}=\mathbf{B}^{\boldsymbol{H}}, \mathbf{C}=\mathbf{C}^{\boldsymbol{H}}$ are Hermitian, there exists $\mu \in \mathbb{R} \cup\{\infty\}$ such that $\mathrm{Q}(\mu)$ is positive definite, and for every fixed $\mathbf{x} \in \mathbb{C}^{n}, \mathbf{x} \neq \mathbf{0}$ the quadratic polynomial (3) has two distinct roots in $\mathbb{R} \cup\{\infty\}$.

Free vibrations of a fluid-solid structures are governed by a non-symmetric eigenvalue problem $[4,8,10,11]$. This problem can be transformed into a definite quadratic eigenvalue problem and it is motivation for further consideration of the definite quadratic pencil and eigenvalue problems for these pencils.

The following Theorem has been proved by Duffin [1]. 
Theorem 1. A Hermitian matrix polynomial $\mathbf{Q}(\lambda)$ is definite if and only if any two (and hence all) of the following properties hold:

- $(\mathbf{x}):=\left(\mathrm{x}^{H} \mathbf{B x}\right)^{2}-\mathbf{4}\left(\mathrm{x}^{H} \mathrm{Ax}\right)\left(\mathrm{x}^{H} \mathrm{Cx}\right)>0$ for every $\mathrm{x} \in \mathbb{C}^{n}, \mathbf{x} \neq \mathbf{0}$

- $\mathbf{Q}(\mu)>0$ for same $\mu \in \mathbb{R} \cup\{\infty\}$

- $\mathbf{Q}(\xi)<0$ for same $\xi \in \mathbb{R} \cup\{\infty\}$.

Without loss of generality, we take that $\xi<\mu$.

$$
\begin{aligned}
& f(\mu ; \mathbf{x})=\mathbf{x}^{H} \mathbf{Q}(\mu) \mathbf{x} \\
& f(\xi ; \mathbf{x})=\mathbf{x}^{H} \mathbf{Q}(\xi) \mathbf{x}
\end{aligned}
$$

where $\mathbf{Q}(\xi)<0<\mathbf{Q}(\mu), \xi<\mu$.

The Rayleigh functional is explicitly given by

$$
p(\mathbf{x}):=\left\{\begin{array}{c}
-\frac{\mathbf{x}^{H} \mathbf{B} \mathbf{x}}{2 \mathbf{x}^{H} \mathbf{A} \mathbf{x}}+\sqrt{\left(\frac{\mathbf{x}^{H} \mathbf{B} \mathbf{x}}{2 \mathbf{x}^{H} \mathbf{A} \mathbf{x}}\right)^{2}-\frac{\mathbf{x}^{H} \mathbf{C} \mathbf{x}}{\mathbf{x}^{H} \mathbf{A} \mathbf{x}}} \text { if } \mathbf{x}^{H} \mathbf{A} \mathbf{x}>0 \\
-\frac{\mathbf{x}^{H} \mathbf{C} \mathbf{x}}{\mathbf{x}^{H} \mathbf{B} \mathbf{x}} \text { if } \mathbf{x}^{H} \mathbf{A} \mathbf{x}=0 \\
-\frac{\mathbf{x}^{H} \mathbf{B} \mathbf{x}}{2 \mathbf{x}^{H} \mathbf{A} \mathbf{x}}-\sqrt{\left(\frac{\mathbf{x}^{H} \mathbf{B} \mathbf{x}}{2 \mathbf{x}^{H} \mathbf{A} \mathbf{x}}\right)^{2}-\frac{\mathbf{x}^{H} \mathbf{C} \mathbf{x}}{\mathbf{x}^{H} \mathbf{A} \mathbf{x}}} \text { if } \mathbf{x}^{H} \mathbf{A} \mathbf{x}<0
\end{array}\right.
$$

The quadratic eigenvalue problem $\mathbf{Q}(\lambda) \mathbf{x}=\mathbf{0}$ has exactly $n$ eigenvalues $\lambda_{1} \leq \cdots \leq \lambda_{n}$ in $(\xi, \mu)$ which satisfy a minmax characterization with respect to $p$, and the safeguarded iterations aiming at $\lambda_{1}$ and $\lambda_{n}$ converge globally and monotonically decreasing and increasing, respectively. Niendorf and Voss [10] gave the following algorithm (Fig.1) for detecting definite quadratic matrix polynomial.

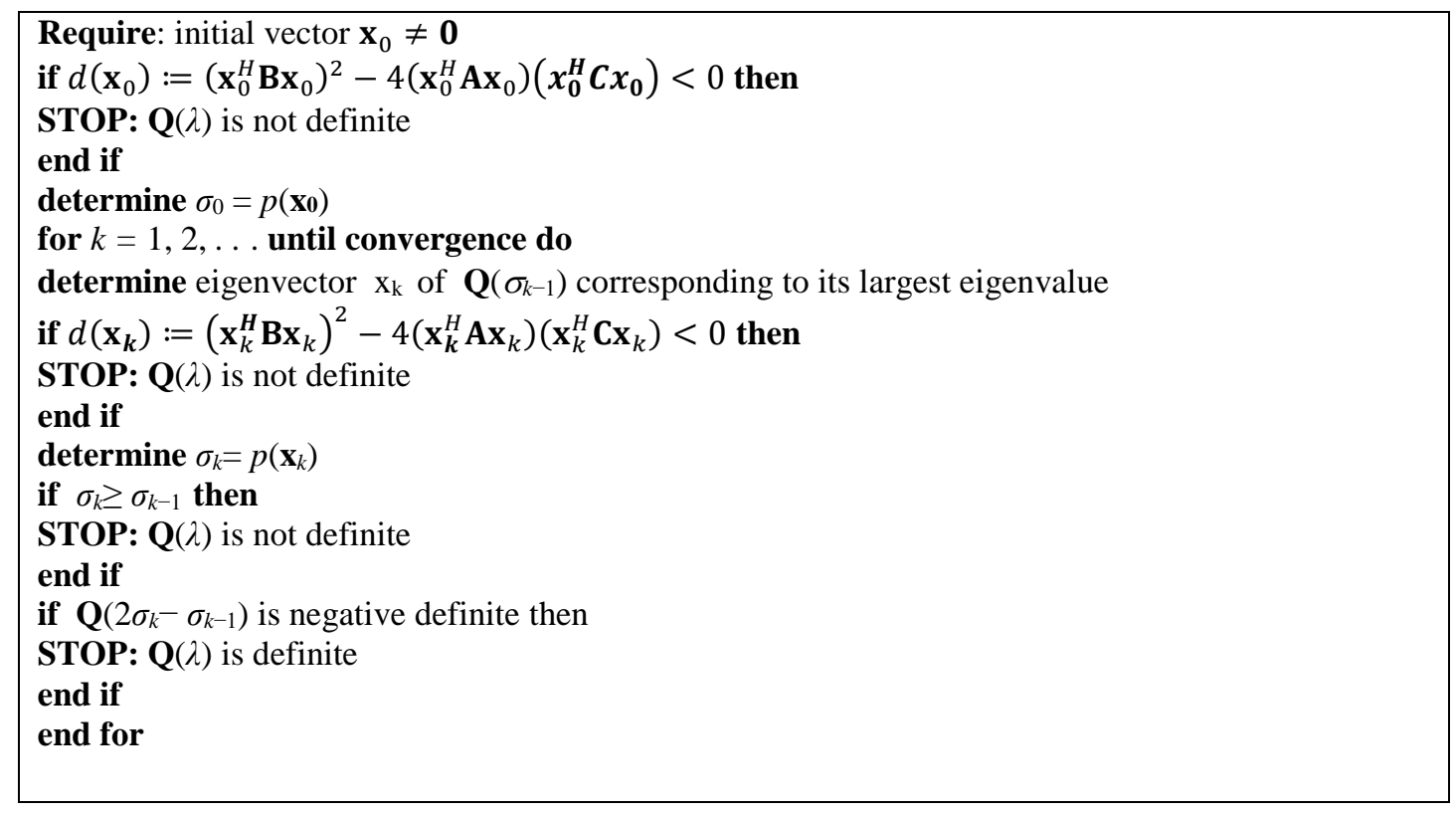

Fig. 1.Algorithm 1.

Now we will briefly explain the Algorithm 1 (Fig.1). If $\mathbf{Q}(\lambda)$ is definite we can determine by Algorithm 1 a parameter $\xi$ such that $\mathbf{Q}(\xi)$ is negative definite. We have to allow for one violation of the monotonicity requirement to incorporate the possible jump of the iterations from one unbounded interval to the other. A second similar sweep aiming at $\lambda_{n}$ discovers a parameter $\mu$ such that $\mathbf{Q}(\mu)>0$. Once parameters $\xi$ and $\mu$ are found such that $\mathbf{Q}(\xi)<0<\mathbf{Q}(\mu)$, the definite linearization can be determined similarly.

Let's analyze the advantages and disadvantages of the Algorithm 1. Its advantages include clarity in the functioning of the algorithm and the ease of programming. The shortcomings of the Algorithm1 are cost of the algorithm which means a large number of flops in every iteration and random selection of the initial vector $\mathbf{x}_{0}$. Let us look first at the cost of the algorithm. 
The $k$-th step of Algorithm 1 requires $n^{3} / 3$ operations for computing 1 Cholesky factorization, $4 n^{2}$ operations for evaluating $\mathbf{Q}\left(2 \sigma_{k}-\sigma_{k-1}\right), 3$ matrix-vector products ( $6 n^{2}$ operations), 3 scalar products ( $6 n$ operations), and the determination of the largest eigenvalue and corresponding eigenvector of a matrix $\mathbf{Q}\left(\sigma_{k-1}\right)$ which seems to be the most expensive part.

Let a quadratic matrix polynomial

$$
\mathbf{Q}(\lambda):=\lambda^{2} \mathbf{A}+\lambda \mathbf{B}+\mathbf{C}, \quad \mathbf{A}=\mathbf{A}^{H}, \quad \mathbf{B}=\mathbf{B}^{H}, \mathbf{C}=\mathbf{C}^{H}
$$

be definite.

Kostic and Sikalo in [8] developed a strategy for targeting of the initial vector $\mathbf{x}$. This leads to reduction in the number of iterations and the algorithm becomes cheaper. Here are the basic theorems on which their strategy is based. Proofs of these theorems are given in [8].

Lemma 1 Problem of the eigenvalues corresponding to the matrix polynomial (5) has 0 as an eigenvalue if and only if $\mathbf{C}$ is singular.

Without loss of generality, Kostic and Sikalo in [8] took that $\xi<\mu$. They considered rank of matrices $\mathbf{A}, \mathbf{B}$ and $\mathbf{C}$.

Let $\operatorname{Rank}(\mathbf{A})=n-p, \operatorname{Rank}(\mathbf{B})=n-q, \operatorname{Rank}(\mathbf{C})=n-r$ where $0<p<n, 0 \leq q \leq n$ and $0<r<n$ and let $\left\{\mathbf{y}_{1}, \mathbf{y}_{2}, \cdots, \mathbf{y}_{n}\right\},\left\{\mathbf{z}_{1}, \mathbf{z}_{2}, \cdots, \mathbf{z}_{n}\right\}$ and $\left\{\mathbf{w}_{1}, \mathbf{w}_{2}, \cdots, \mathbf{w}_{n}\right\}$ be orthonormal base of vector space $\mathbb{C}^{n}$ consisting of eigenvectors of the corresponding matrix $\mathbf{A}, \mathbf{B}$ and $\mathbf{C}$ respectively. Without loss of generality, Kostic and Sikalo in [8] took

$$
\mathbf{A y}_{i}=0 i=1,2, \cdots, p, \quad \mathbf{B z}_{j}=0, j=1,2, \cdots, q, \quad \mathbf{C w}_{k}=0 \quad k=1,2, \cdots, r .
$$

Theorem 2 Let $\mathbf{Q}(\xi)<0<\mathbf{Q}(\mu)$ and $\xi<\mu$ then stands $\mu>a_{1}>c_{1}>\xi$ where

$$
a_{1}:=\max \left\{-\frac{\mathbf{y}^{H} \mathbf{C y}}{\mathbf{y}^{H} \mathbf{B y}}: \mathbf{y} \in\left\{\mathbf{y}_{1}, \mathbf{y}_{2}, \cdots, \mathbf{y}_{p}\right\}\right\} \text { and } c_{1}:=\min \left\{-\frac{\mathbf{y}^{H} \mathbf{C y}}{\mathbf{y}^{H} \mathbf{B y}}: \mathbf{y} \in\left\{\mathbf{y}_{1}, \mathbf{y}_{2}, \cdots, \mathbf{y}_{p}\right\}\right\} \text {. }
$$

Theorem 3 Let $\mathbf{Q}(\xi)<0<\mathbf{Q}(\mu)$ and $\xi<\mu$ then stands $\mu>a_{2}$ and $c_{2}>\xi$ where

$$
\begin{aligned}
& a_{2}:=\max \left\{-\frac{\mathbf{w}^{H} \mathbf{B w}}{2 \mathbf{w}^{H} \mathbf{A w}}: \mathbf{w} \in\left\{\mathbf{w}_{1}, \mathbf{w}_{2}, \cdots, \mathbf{w}_{r}\right\} \text { and } \mathbf{w}^{H} \mathbf{A} \mathbf{w}>0\right\} \\
& c_{2}:=\min \left\{-\frac{\mathbf{w}^{H} \mathbf{B w}}{2 \mathbf{w}^{H} \mathbf{A} \mathbf{w}}: \mathbf{w} \in\left\{\mathbf{w}_{1}, \mathbf{w}_{2}, \cdots, \mathbf{w}_{r}\right\} \text { and } \mathbf{w}^{H} \mathbf{A} \mathbf{w}<0\right\}
\end{aligned}
$$

Theorem 4 Let $\mathbf{Q}(\xi)<0<\mathbf{Q}(\mu), \xi<\mu$ and $a:=\max \left(a_{1}, a_{2}\right)$ and $c:=\min \left(c_{1}, c_{2}\right)$ then applies $\mu>a$ and $c>\xi$ where $a_{1}$, $a_{2}, c_{1}$ and $c_{2}$ are defined as in Theorem 2 and Theorem 3 .

If we now apply Algorithm 1 to determine the parameter $\mu$ for the initial vector $\mathbf{x}_{0}$ we take the vector through which we specify $a$. Analogously if we apply Algorithm 1 (Fig. 1) for determining parameter $\xi$ for initial vector $\mathbf{x}_{0}$ we take the vector through which we have determined $c$.

There remains to consider what happens in the case of the singularity of the matrix $\mathbf{B}$.

Theorem 5 Let $\mathbf{Q}(\xi)<0<\mathbf{Q}(\mu), \xi<\mu$, $\operatorname{Rank}(\mathbf{B})=n-q$ and $q>0$ than $\mu>0$ or $\xi<0$.

Remark: In the proof of the Theorem 5 [8] it is clear that $\mathbf{z}^{H} \mathbf{A z}$ has the same sign for all $\mathbf{z} \in\left\{\mathbf{z}_{1}, \mathbf{z}_{2}, \cdots, \mathbf{z}_{q}\right\}$. If $\mathbf{z}^{H} \mathbf{A z}>$ 0 we can multiply problem (5) by -1 and get a new definite eigenvalue problem.

$$
\mathbf{Q}(\lambda):=\lambda^{2} \mathbf{A}_{1}+\lambda \mathbf{B}_{1}+\mathbf{C}_{1}, \quad \mathbf{A}_{1}=-\mathbf{A}, \quad \mathbf{B}_{1}=-\mathbf{B}, \quad \mathbf{C}_{1}=-\mathbf{C}
$$

Problem (7) has the same eigenvalues as problem (5), but with $\mathbf{z}^{H} \mathbf{A}_{1} \mathbf{z}<0$ and $\xi<0$. Therefore, in the case of singularity of the matrix $\mathbf{B}$ without loss of generality we can consider that $\mathbf{z}^{H} \mathbf{A z}<\mathbf{0}$ and $\xi<0$. In the case of singularity of the matrix $\mathbf{B}$ in order to reduce the costs of determining the initial vector $\mathbf{x}_{0}$ it is taken $\mathbf{x}_{0}=\mathbf{z}_{1}$.

\section{The effective algorithm for determining the initial vector}

Kostic and Sikalo in [8] have used some properties of the matrix $\mathbf{A}, \mathbf{B}$ and $\mathbf{C}$ for determination of the initial vector $\mathbf{x}_{0 .}$ Those properties are for example ranks of the matrices, eigenvectors belonging to the eigenvalue zero of the $\mathbf{A}, \mathbf{B}$ and $\mathbf{C}$ 
matrices. For the implementation of this strategy they would have to find eigenspace of the matrices $\mathbf{A}, \mathbf{B}$ and $\mathbf{C}$, which are associated with the eigenvalue zero.

The basic idea of this paper is still using nonzero eigenvalues of matrices $\mathbf{A}$ and $\mathbf{B}$ to get a better location parameters and better initial vector. The acceleration of the algorithm is also obtained by improvement of the initial vector $\mathbf{x}_{0}$. Our aim is getting high-quality algorithm for the determination of the initial vector. This algorithm is obtained by using results of the paper [8] and this paper.

In order to accelerate Algorithm 1 we consider a better localization of the parameters $\mu$ and $\xi$ which hold $\mathbf{Q}(\mu)>0>$ $\mathbf{Q}(\xi)$ and $\mu>\boldsymbol{\xi}$. From this localization we will get the initial vector $\mathbf{x}_{0}$.

In [8] is noticed that matrices $\mathbf{A}$ and $\mathbf{C}$ must be singular. The singularity of matrix $\mathbf{B}$ can but doesn't have to be satisfied. If matrix $\mathbf{B}$ is singular than considering Remark for the initial vector we take $\mathbf{x}_{0}=\mathbf{z}_{1}$. If the matrix $\mathbf{B}$ is not singular theorems 2, 3 and 4 give us possibility of choosing initial vector $\mathbf{x}_{\mathbf{0}}$. However this means we must determine all eigenvectors of the matrix $\mathbf{A}$ that belong to eigenvalue zero and all eigenvalues of the matrix $\mathbf{C}$ that belong to eigenvalue zero. Also, this procedure is weighted by finding a large number of products of vectors and matrices and scalar products. This brings additional algorithm costs (a large number of flops and long time computing). To avoid the large number of flops in the case when matrix $\mathbf{B}$ is not singular, we consider a new strategy.

We assume that the matrix $\mathbf{A}$ has at least one positive and at least one negative eigenvalue. We denote the largest eigenvalue of matrix $\mathbf{B}$ with $\lambda_{n} \mathbf{B}$ and the corresponding eigenvector with $\mathbf{q} 1$. We denote the smallest eigenvalue of matrix A with $\lambda_{1 \mathrm{~A}}$ and the corresponding eigenvector with $\mathbf{f}_{\mathbf{n}}$. It is clear that $\lambda_{1 \mathrm{~A}}<0$.

For every fixed $\mathbf{x} \in \mathbb{C}^{n}, \mathbf{x} \neq \mathbf{0}$ is

$$
\begin{aligned}
& \mu^{2} \mathbf{x}^{H} \mathbf{A} \mathbf{x}+\mu \mathbf{x}^{H} \mathbf{B} \mathbf{x}+\mathbf{x}^{H} \mathbf{C} \mathbf{x}>0 \\
& \xi^{2} \mathbf{x}^{H} \boldsymbol{A} \mathbf{x}+\xi \mathbf{x}^{H} \mathbf{B} \mathbf{x}+\mathbf{x}^{H} \mathbf{C} \mathbf{x}<0
\end{aligned}
$$

Subtracting the inequality (9) from inequality (8) yields

$$
(\mu-\xi)(\mu+\xi) \mathbf{x}^{H} \mathbf{A} \mathbf{x}+(\mu-\xi) \mathbf{x}^{H} \mathbf{B} \mathbf{x}>0 \stackrel{\xi<\mu}{\Longrightarrow}(\mu+\xi) \mathbf{x}^{H} \mathbf{A} \mathbf{x}+\mathbf{x}^{H} \mathbf{B} \mathbf{x}>0 .
$$

Further we get

$$
(\mu+\xi) \frac{\mathbf{x}^{\mathrm{H}} \mathbf{A} \mathbf{x}}{\mathbf{x}^{\mathbf{H}} \mathbf{x}}+\lambda_{n \mathbf{B}}>(\mu+\xi) \frac{\mathbf{x}^{\mathrm{H}} \mathbf{A} \mathbf{x}}{\mathbf{x}^{\mathrm{H}} \mathbf{x}}+\frac{\mathbf{x}^{\mathrm{H}} \mathbf{B} \mathbf{x}}{\mathbf{x}^{\mathrm{H}} \mathbf{X}}>0
$$

From (11) we get for every fixed $\mathbf{x} \in \mathbb{C}^{n}, \mathbf{x} \neq \mathbf{0}$

$$
(\mu+\xi) \frac{\mathbf{x}^{\mathbf{H}} \mathbf{A x}}{\mathbf{x}^{\mathbf{H}} \mathbf{X}}>-\lambda_{n \mathbf{B}}
$$

Specially for $\mathbf{x}=\mathbf{q}_{\mathbf{1}}$ we get

$$
(\mu+\xi) \frac{\mathbf{q}_{\mathbf{1}}{ }^{\mathbf{H}} \mathbf{A q _ { 1 }}}{\mathbf{q}_{\mathbf{1}}{ }^{\mathrm{H}} \mathbf{q}_{\mathbf{1}}}>-\lambda_{n \mathbf{B}}
$$

Since $\mathbf{q}_{1}$ is eigenvector of eigenvalue $\lambda_{1 \mathbf{A}}<0$ from (13) is obtained

$$
(\mu+\xi) \lambda_{1 \mathrm{~A}}>-\lambda_{n \mathrm{~B}}
$$

From (14) and $\lambda_{1 \mathrm{~A}}<0$ we get

$$
\mu+\xi<-\frac{\lambda_{n \mathbf{B}}}{\lambda_{1 \mathbf{A}}}
$$

From (14) and $\mu-\xi>0$ we get

$$
\xi<-\frac{\lambda_{n \mathbf{B}}}{2 \lambda_{1 \mathrm{~A}}}
$$

This way we get upper bound for parameter $\xi$ in the case where $\mathbf{B}$ is not singular. In this case because of the great significance of the matrix $B$ for definite eigenvalue problem we will take $\mathbf{x}_{0}=0.9 \mathbf{q}_{1}+0.1 \mathbf{f}_{\mathbf{n}}$ for the initial vector. Now we will give an effective algorithm for determination of the initial vector $\mathbf{x}_{0}$. 
Examine singularity of matrix $\mathbf{B}$

If matrix $B$ is singular then $\mathbf{x}_{0}=\mathbf{z}_{1}$

else

determine smallest eigenvalue of matrix $\mathbf{A} \lambda_{\mathbf{1 A}}$ and corresponding eigenvector $\mathbf{q}_{\mathbf{1}}$

determine largest eigenvalue of matrix $\mathbf{B} \lambda_{\mathbf{n B}}$ and corresponding eigenvector $\mathbf{f}_{\mathbf{n}}$

$\mathbf{x}_{0}=0.9 \mathbf{q}_{\mathbf{1}}+0.1 \mathbf{f}_{\mathbf{n}}$

end if

Fig.2. Algorithm 2

Fig.2. gives the effective algorithm for determining of the initial vector $\mathbf{x}_{0}$. This algorithm is based on the structure of the definite quadratic eigenvalue problem. At the end we would like to point out the following. To determine whether the matrix $\mathbf{B}$ is singular we have to decomposition this matrix. From this decomposition we can see all the eigenvalues and the corresponding eigenvectors of matrix $\mathbf{B}$. Therefore, if the matrix $\mathbf{B}$ is not singular, we already have an eigenvector belonging to the largest eigenvalue at no additional cost.

The following two examples numerically show justification for applying Algorithm 2.

Example 1

In the quadratic matrix polynomial $\mathbf{Q}(\lambda):=\lambda^{2} \mathbf{A}+\lambda \mathbf{B}+\mathbf{C}$ we will take

$$
\mathbf{A}=\left(\begin{array}{lll}
1 & 2 & 3 \\
2 & 5 & 6 \\
3 & 6 & 8
\end{array}\right), \mathbf{B}=\left(\begin{array}{ccc}
0 & 0 & 0 \\
0 & 10^{9} & 0 \\
0 & 0 & -10^{15}
\end{array}\right) \text { and } \mathbf{C}=\left(\begin{array}{lll}
4 & 0 & 0 \\
0 & 5 & 9 \\
0 & 9 & 5
\end{array}\right)
$$

We tested the speed of determination weather the matrix is or is not definite. First, we used only with Algorithm 1. Numerical tests were performed by using MATLAB. The initial vector is determined by using function randn. We did 100 tests and we obtained that average number of expensive iterations for getting the answer is 4.62. When we use Algorithm 1 and Algorithm 2 we get the answer immediately in the preparatory phase of the Algorithm 1. Therefore, the answer is obtained without expensive iterations.

Example 2

In the quadratic matrix polynomial $\mathbf{Q}(\lambda):=\lambda^{2} \mathbf{A}+\lambda \mathbf{B}+\mathbf{C}$ we will take

$$
\mathbf{A}=\left(\begin{array}{lll}
1 & 2 & 3 \\
2 & 5 & 6 \\
3 & 6 & 8
\end{array}\right), \mathbf{B}=\left(\begin{array}{ccc}
-10^{9} & 0 & 0 \\
0 & -10^{9} & 0 \\
0 & 0 & 1
\end{array}\right) \text { and } \mathbf{C}=\left(\begin{array}{lll}
4 & 0 & 0 \\
0 & 5 & 9 \\
0 & 9 & 5
\end{array}\right)
$$

Once again we did numerical experiment with MATLAB. The initial vector was determined by using function randn. We did 100 tests and we obtained that the average number of expensive iterations for getting the answer is 4.17 . When we used Algorithm 1 and Algorithm 2 we obtained the answer after one expensive iteration.

Example 1 and Example 2 show the importance of using Algorithm 1 for determination of the initial vector. This is especially important when matrix $\mathbf{B}$ is singular or when there exists great difference between absolute value of the biggest and the absolute value of the smallest eigenvalue as in Example 2.

\section{Conclusion}

This paper gives the effective algorithm for the determination of the initial vector $\mathbf{x}_{0}$. If the initial vector $\mathbf{x}_{0}$ is better determined we get the algorithm acceleration. In previous papers there were attempts of getting a better initial vector $\mathbf{x}_{0}$ that were based on the exploitation of some properties of the matrices $\mathbf{A}, \mathbf{B}$ and $\mathbf{C}$. Previously used properties were ranks of matrices and their eigenvectors which belong to eigenvalue zero. In this paper we also give exploitation of the eigenvector $\mathbf{q}_{\mathbf{1}}$ which is corresponding vector of the eigenvalue $\lambda_{\mathbf{1 A}}$ of matrix $\mathbf{A}$. We also give exploitation of the eigenvector $\mathbf{f}_{\mathbf{n}}$ which is corresponding vector of the eigenvalue $\lambda_{\mathbf{n B}}$ of matrix $\mathbf{B}$. In Example 1 and Example 2 we have concretely shown the justification for applying the Algorithm 2.

Therefore, in this paper we sum up results from previous papers with the results of this paper. This way we get a highquality algorithm for determining initial vector $\mathbf{x}_{0}$. This algorithm enables significant acceleration of the Algorithm 1 . The further research will go in the direction of the use of features of the matrix $\mathbf{A}, \mathbf{B}$ and $\mathbf{C}$, which determine square pencil that is used for improvement of localization of the parameters $\xi$ and $\mu$. 


\section{References}

[1] Duffin, R. J. (1955). A minimax theory for overdamped networks, J. Rat. Mech. Anal., 4 (1955) 221-233

[2] Guo, C.-H.; Higham, N.J. \& Tisseur, F. (2007). Detecting and solving hyperbolic quadratic eigenvalue problems, Technical Report 117, The Manchester Institute of Mathematical Sciences, The University of Manchester, (2007)

[3] Guo, C.-H. \& Lancaster P. (2005). Algorithms for hyperbolic quadratic eigenvalue problems, Math.Comp., 74 (2005) 1777-1791, ISSN 0025-5718

[4] Harris, C. \& Piersol, A. (2002). Harris Shock and Vibration Handbook, 5 ed., McGraw-Hill, NewYork, 2002

[5] Higham, N. J.; Tisseur, F.\& Van Dooren, P.M. (2002). Detecting a definite Hermitian pair anda hyperbolic or elliptic quadratic eigenvalue problem, and associated nearness problems, Linear Algebra Appl., (2002) 351-352 455-474, ISSN 0024-3795

[6] Higham, N.; Mackey, D. \& Tisseur, F. (2009). Definite matrix polynomials and their linearization by definite pencils, SIAM J. Matrix Anal. Appl. 33 (2009) 478-502, ISSN 0895-4798

[7] Kostic, A. (2016). Eigenvalue Problems In: Applied linear algebra in action, Katsikis, N. (Ed.),57-83, InTech ISBN 978-953-51-2419-1, Rijeka

[8] Kostic, A. \&. Sikalo, S. (2015). Definite Quadratic Eigenvalue Problems. Procedia Engineering 100 (2015) 56 - 63, ISSN $1877-7058$

[9] Kostic, A. \& Voss, H. (2013). On Sylvester's law of inertia for nonlinear eigenvalue problems, Electr. Trans. Numer. Anal., 40 (2013) 82-93, ISSN 1068-9613

[10] Niendorf, V. \& Voss, H. (2010). Detecting hyperbolic and definite matrix polynomials, Linear Algebra Appl., 432 (2010) 1017-1035, ISSN 0024-3795

[11] Stammberger, M. \& Voss, H. (2010). On an unsymmetric eigenvalue problem governing free vibrations of fluidsolid structures, Electr. Trans. Numer. Anal., 36 (2010), 113-125, ISSN 1068-9613

[12] Tisseur, F. \& Meerbergen, K. (2001). The quadratic eigenvalue problem. SIAM Review, 43 (2001) 235-286, ISSN 0036-1445 